\title{
Minimax Optimization of Reactance Connected in Series on Capacitor to Eliminate Resonance
}

\author{
Pu Deng ${ }^{1, a}$, Xiao-bo Liu ${ }^{2, b}$, Xian-song Pi ${ }^{1, a}$, Hong-li Li ${ }^{3, c}$, Feng-yuan Wang ${ }^{1, a}$ \\ ${ }^{1}$ Guizhou power grid company power grid planning and research center, Guiyang, China \\ ${ }^{2}$ Guizhou University, Guizhou Province, China \\ ${ }^{3}$ Duyun Electric Power Bureau, Duyun, Guizhou Province, China \\ ae-mail:2410406553@qq.com, ${ }^{b}$-mail:799797284@qq.com
}

\begin{abstract}
In order to solve the problem of harmonic resonance in shunt capacitor at $220 \mathrm{kV}$ or $110 \mathrm{kV}$ transformer substation, use the PSCAD software to construct harmonic impedance models of capacitor, transformer, load, short impedance and harmonic source, give resonance objective function such as harmonic voltage amplification factor on load bus, etc. With minimax optimization, the maximum values of objective function in all the power grid parameter space are minimized by changing reactance rate. The conclusion is: resonance is eliminated with best effect and generality by the rate $12 \%$; resonance is eliminated with second effect by rate $4.3 \% \sim 4.5 \%$, but the rate $4.3 \% \sim 4.5 \%$ has better technical and economic value and Suitable for most of $220 \mathrm{kV}$ and $110 \mathrm{kV}$ substation.
\end{abstract}

KEYWORD: PSCAD; Harmonic amplification; Reactance ratio; Harmonic impedance; Minimax optimization

\section{INSTRUCTION}

Shunt power capacitors are prone to produce the harmonic resonance in larger harmonic wave of the power grid, which damages parallel capacitor, amplifies public access points (PCC) harmonic voltage, and deteriorates grid power quality.

Harmonic resonance of capacitor is the result of two factors working together: one is the capacitor impedance and grid impedance exist with that the resonance frequency consistent with integer harmonic frequency phase; second, the substation injected with larger harmonic component consistent with the resonance frequency.

Capacitor in series a reactance, can avoid the resonant. Reactance rate of capacitor banks are made up of cell capacity and series reactive element .That is ratio of series reactance inductance and capacitive reactance of capacitor, and it's the key factor to prevent the capacitor resonance.

For the problems of reactance rate, they have had a lot of analysis and research both at home and abroad $^{[1-5]}$.

Before the design of capacitor, literature and standard need to measure the background harmonic at substation capacitors access point, this is hard work in the design of the new substation capacitors ${ }^{[6-}$ 13]

To solve the selection problem of reactance rate of new substation capacitor design, we need to study the general properties for the ability of the reactance rate to eliminate harmonic, contains study power universal properties under the conditions of various factors. Thus, this article aim at resonance problem of the substation parallel capacitor of the $220 \mathrm{kV}$ and $110 \mathrm{kV}$, build a model of parallel capacitor group, transformer, load, power grid short-circuit impedance and harmonic source, and analyze the scope of the model parameters, propose the objective function which reflect the resonant level and the harm degree of capacitor group. In all the power grid state space of model, for target function minimal optimization about maximum of reactance rate, it is concluded that the universal significance of optimizing the reactance rate.

\section{CIRCUIT, MODELS AND PARAMETERS}

\subsection{Resonant circuit}

According to the typical equipment and electrical wiring of $220 \mathrm{kV} \sim 110 \mathrm{kV}$ substation, use the PSCAD software to build the substation equivalence resonant circuit includes capacitor, transformer, load and power grid short-circuit impedance. The type of transformer contains three windings and double windings. Capacitor inject to low voltage side of the main transformer, the load bus of three-winding transformer is medium voltage side bus, and the load bus of double-winding transformer is a low voltage side bus. Harmonic sources adopts the constant current source model ${ }^{[14 \sim 16]}$ that is the background 
harmonic inserted from the high voltage side bus of main transformer or the non-linear load harmonic inserting load bus. Thus four harmonic equivalent circuit are formed, as shown in fig. 1.

\subsection{Harmonic impedance model and parameter range}

Resonant circuit contains short circuit impedance, impedance transformer, capacitor banks impedance and load impedance, which are considered as pure resistance, pure capacitance or pure inductance, ignorance the skin effect of electrical components ${ }^{[17]}$. Capacitive harmonic impedance is the impedance of fundamental wave divided by the harmonic frequency, while inductive harmonic impedance is the impedance of fundamental wave multiplied by the harmonic frequency.

To adopting the benchmark as follow for the MAO value transform:

Benchmark capacity: the actual operating capacity Q of capacitor group.

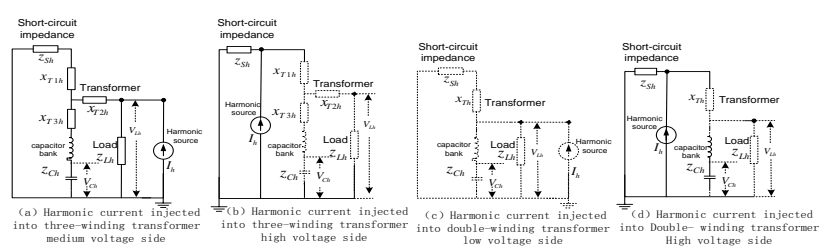

Fig. 1 Harmonic injection mode and resonance equivalent circuit

Benchmark voltage: the operating voltage of main transformer branch point

In Fig.1, the MAO value of each harmonic impedance is derived as follows.

(1)the harmonic impedance of the system shortcircuit impedance

$$
Z_{S h}=\frac{0.15}{\sqrt{0.15^{2}+1}} \frac{\left(Q / S_{T}\right)}{\left(S / S_{T}\right)}+j \frac{h}{\sqrt{0.15^{2}+1}} \frac{\left(Q / S_{T}\right)}{\left(S / S_{T}\right)} .
$$

The number, 0.15 ,is the ratio of the resistance and inductance of short-circuit impedance, which has little impact on resonant capacitor.

$h$ is harmonic frequency.

$S / S T$ is transformer short-circuit ratio in which $S$ is short-circuit capacity of main transformer high voltage side and $S T$ is transformer capacity. In transformer substation, $\mathrm{n}$ transformers can be equivalent to one, and the equivalent capacity is $n \times S T$. After a large number of data analysis, $S / S T$ value of the $220 \mathrm{kV}$ and $110 \mathrm{kV}$ substation is between 3.6 100.

$Q / S T$ is the actual running capacitor compensation rate. According to the guidelines for the power system voltage and reactive power technology ${ }^{[19]}$, compensation rate of substation capacitor should be less than 0.3 . So $Q / S T$ is among $0 \sim 0.3$.

(2)harmonic reactance of three-winding transformer

$$
\begin{gathered}
X_{T 1 h}=j \cdot 0.14 \cdot \delta \cdot h \cdot Q / S_{T} . \\
X_{T 2 h}=j \cdot(-0.007) \cdot \delta \cdot h \cdot Q / S_{T} \\
X_{T 3 h}=j \cdot 0.09 \cdot \delta \cdot h \cdot Q / S_{T} .
\end{gathered}
$$

The constant $0.14,0.007,0.09$ in formula are typical values of impedance voltage of high, medium and low three windings. $\delta$ is the range of transformer impedance. Impedance voltage of different $220 \mathrm{kV}$ and $110 \mathrm{kV}$ substation transformers are different, each transformer tap access will also affect the actual impedance, and $\delta$ is in the range of $0.7 \sim 1.3$ times of typical values.

(3)double-winding transformer harmonic reactance

$$
X_{T h}=j \cdot 0.14 \cdot \delta \cdot h \cdot Q / S_{T} .
$$

The constant 0.14 is typical value of the doublewinding transformer impedance voltage, $\delta$ is the transformer impedance variation range as described above.

(4)the harmonic impedance of capacitor group

$$
Z_{c h}=j \cdot(x h-(1 / h))
$$

The $x$ is series reactance rate of capacitor, and studied in the range of $0 \sim 0.13$.

(5)load harmonic impedance

$$
\begin{gathered}
R_{L}=Q / S_{T} /(\cos \phi \cdot \gamma) \cdot \\
X_{L h}=j \cdot Q / S_{T} /\left(\sqrt{1-\cos ^{2} \phi} \cdot \gamma\right) . \\
Z_{L h}=\left(R_{L} \cdot X_{L h}\right) /\left(R_{L}+X_{L h}\right) .
\end{gathered}
$$

$\gamma$ is the main transformer load rate that reflects the size of the load. That is the ratio of load capacity and the main transformer capacity, and the range is $0.1 \sim 1.1 . \cos \phi$ is the load power factor with the range of $0.8 \sim 1$.

(6)the current harmonic model of the harmonic source

$$
I_{h}=I_{1} \cdot a_{h} .
$$

$I_{1}$ is the fundamental wave current of nonlinear load, and ah is the harmonic source of $h$ times harmonic current content, all values of ah are in the range of $0 \sim 1$.

(7)Model variables

The reactance rate $x$ is the control variable to solve equation. The rest of the variable is the state 
parameters of the power grid, and the state variables can be made up the state vector $\dot{z}$.

$$
\begin{gathered}
\dot{\mathrm{z}}=\left(\delta, \gamma, S / S_{\mathrm{T}}, Q / S_{\mathrm{T}}, \cos \phi, a_{2}, a_{3, \ldots,} a_{\mathrm{n}}\right) . \\
\dot{\mathrm{z}} \in D . \\
D=\left\{\begin{array}{l}
\left(\delta, \gamma, S / S_{T}, Q / S_{T}, \cos \phi, a_{2}, a_{3}, \ldots a_{n}\right) \\
0.7 \leq \delta \leq 1.3, \quad 0.1 \leq \gamma \leq 1, \quad 3 \leq S / S_{T} \leq 100 \\
0 \leq Q / S_{T} \leq 0.3,0.8 \leq \cos \phi \leq 1,0 \leq a_{2}, a_{3}, \ldots a_{n} \leq 1
\end{array}\right)
\end{gathered}
$$

Each variable, which can be made up the vector, has a certain value range, and $\dot{z}$ is certain in its power grid state space.

\section{THE OBJECTIVE FUNCTION OF REFLECTING THE CAPACITOR RESONANT LEVEL}

\subsection{The magnification $(\lambda)$ of voltage total harmonic distortion rate of load bus}

$\lambda$ is the ratio of the load bus voltage total harmonic distortion rate of metering capacitor resonant and regardless of the capacitor resonant (capacitor is not put in), measure deteriorated degree of power quality of harmonic at the point of common coupling (PCC), cause by the capacitor resonant.

$$
\lambda(x, \dot{z})=T H D_{L} / \lim _{Q / S_{T} \rightarrow 0}\left(T H D_{L}\right) .
$$

$T H D_{L}$ is the total harmonic distortion rate of load bus voltage

$$
T H D_{L}=\sqrt{\sum_{h} V_{L h}^{2}} / V_{1}
$$

$V_{l}$ is the fundamental wave voltage of load bus, $V_{L h}$ is h times harmonic voltage of load bus, as shown in fig.1. the analysis formula of $V_{L h}$ is easily derived from the source of harmonic current, short-circuit impedance, transformer impedance, reactance of capacitor banks and load impedance.

The dual effects of $T H D_{L}$ with harmonic source and the capacitor resonant factors(the match of the impedance parameters).

Capacitor reactance parameters are difficult to cooperate with the system parameters in the situation that capacitor input is small and the resonant degree is very small. Under the limit state that capacitor compensation rate $Q / S_{\mathrm{T}} \rightarrow 0$, resonant capacitor will not occur. So value of $\lim _{Q / S_{T} \rightarrow 0}\left(T H D_{L}\right)$ only because of the influence of harmonic sources, and not affected by capacitor resonant.

Analytic expression of $\lambda$ is lengthy, there are four kinds of circuit in fig.1, the appendix A shows the analytic expression of $\lambda$.

Notice that the formula (15) the fundamental wave voltage of load bus $V_{l}$ and nonlinear load current fundamental wave current $I_{1}$ are reduction of a fraction to eliminate, $\lambda$ is the multivariate function consists of the independent variable $(\mathrm{x}, \dot{\mathrm{z}})$.

\subsection{Voltage total harmonic distortion rate magnification $(\mu)$ of Capacitor element}

$\mu$ is the ratio of the load bus voltage total harmonic distortion rate of metering capacitor resonant and regardless of the capacitor resonant (capacitor is not put in), measure the effect of the resonance to capacitor group overvoltage, and reflect the harm degree of capacitor group to their own equipment.

$$
\mu(x, \dot{z})=T H D_{C} / \lim _{Q / s_{r} \rightarrow 0}\left(T H D_{C}\right)^{\circ}
$$

Among them

$$
T H D_{c}=\sqrt{\sum_{h} V_{c h}^{2}} / V_{c 1} \cdot
$$

$T H D_{C}$ as voltage total harmonic distortion rate on the capacitor component. $\mathrm{Vc1}$ is fundamental voltage on the capacitor element, $V_{c h}$ is the h times harmonic voltage on the capacitor element, as shown in fig.1.

The dual effects of $T H D_{C}$ with harmonic source and the capacitor resonant factors(the match of the impedance parameters).

$\lim _{Q / S_{\tau} \rightarrow 0}\left(T H D_{C}\right)$ is the voltage total harmonic distortion rate of capacitor element when disregarding capacitor resonant, its limitation exist and easily derived.

The analytic expression of $\mu$ and its derivation are shown in appendix B. From the formula (17), the fundamental wave voltage $V_{c l}$ of capacitor element and fundamental wave current $I_{1}$ of nonlinear load is reduction of a fraction, each $\mu$ of circuit in fig. 2 is the multivariate function consists of independent $\operatorname{variable}(x, \dot{\mathrm{z}})$.

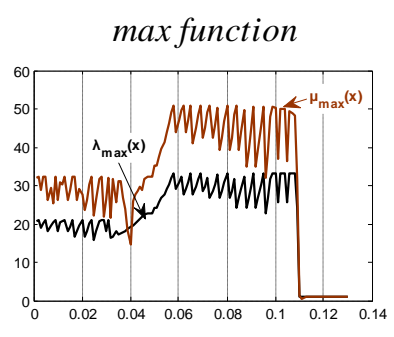

reactance rate $x$

(a) Harmonic current injected into three-winding transformer medium voltage side

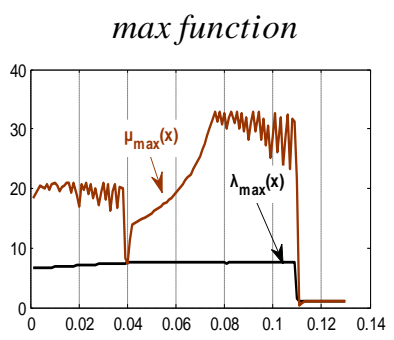

reactance rate $x$

(b) Harmonic current injected into three-winding transformer high voltage side 


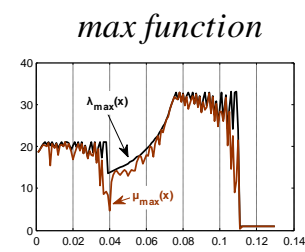

reactance rate $x$

(c) Harmonic current injected into doublewinding transformer low voltage side

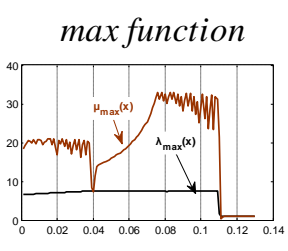

reactance rate $x$

(d) Harmonic current injected into double-winding transformer high voltage side
Fig. 2 Curves of objective maximum functions

According to the nominal voltage $1000 v$ ac power system using parallel capacitor part 1: general rules ${ }^{[18]}$, long time working voltage of capacitor should satisfy $V_{\mathrm{C}} \leq 1.1 V_{\mathrm{N}}, V_{C}$ is capacitor working voltage, is the root-mean-square value of ac voltage, $V_{N}$ is rating voltage designed for capacitor. According to the guidelines for the power system voltage and reactive power technology ${ }^{[19]}$, voltage deviation of $35 \mathrm{kV}$ should be within $-3 \% \sim 7 \%$, voltage deviation of $10 \mathrm{kV}$ should be within $\pm 7 \%$, the highest capacitor voltage deviation is 1.07 .

According to the above prescribed standards, accounting the voltage total harmonic distortion rate $T H D_{C}$ which capacitor element can withstand.

$$
\begin{aligned}
& \sqrt{1.0^{2} 7+(T H Q \cdot 1.07} \leq 1 . \\
T H D_{C} & =23.85 \%
\end{aligned}
$$

Harmonics in public supply network standard ${ }^{[20]}$, the voltage total harmonic distortion rate of $35 \mathrm{kV} \sim$ $10 \mathrm{kV}$ bus which accessed by capacitor banks should be less than $5 \%$.Because $23.85 \%$ is 4.77 times that of $5 \%$,in the case of substation harmonic power quality qualified, if the capacitor element total harmonic distortion rate $\mu$ is less than 4.77 times, it's guaranteed for capacitor element to the safe operation for a long time.

\section{MINIMAX OPTIMIZATION OF OBJECTIVE FUNCTION}

\subsection{The maximum objective function about} reactance rate $x$

A certain value of control variable $x$, here exit a vector $\dot{z}$ in the state space $D$, which can lead $\lambda(x, \dot{z})$ has a max value, the mapping form of $x$ and the maximum value make up target function $\lambda(x, \dot{z})$.

$$
\lambda_{\max }(x)=\max _{\dot{z} \in D}[\lambda(x, \dot{z})] .
$$

$\lambda(x, \dot{z})$ is a single value function by regarding $x$ as the independent variable.

Similarly, to define the maximum function of $\square$ max $(x)$ about reactance rate $x$

$$
\mu_{\max }(x)=\max _{\dot{z} \in D}[\mu(x, \dot{z})] .
$$

\subsection{Minimax optimization of objective function.}

The minimax optimization of objective function $\max _{\text {max }}(x)$ with respect to $x$ is expressed as:

$$
\min _{x}\left[\lambda_{\max }(x)\right] \text { that is } \min _{x}\left[\max _{\dot{z} \in D} \lambda(x, \dot{z})\right]
$$

Optimal explanation is: after optimized by series reactance rate $x$, the max value of objective function reaches to the minimize in the whole power grid state space.

Similarly, the minimax optimization of objective function $\mu(x, \dot{z})$ with respect to $x$ is expressed as:

$$
\min _{x}\left[\mu_{\max }(x)\right] \text { that is } \min _{x}\left[\max _{\dot{z} \in D} \mu(x, \dot{z})\right]
$$

\subsection{The numerical solution of the minimax optimization}

$\lambda$ and $\mu$ are continuous and differentiable, but they are not convex functions in the multi-dimensional grid state space D. To solve the maximum, grid method is commonly used in engineering numerical calculation.

The harmonic content of even harmonics 4 times or more and harmonics 21 or more in the power grid is small, which can be neglected. Therefore, this article only consider harmonic content a that have larger influence on capacitor resonant ,such as 2, 3, 5, 7, 9, 11, 13, 15, 17 and 19 times harmonic content. So, the state vector $\dot{z}$ can be simplified to 15 variables.

$$
\begin{gathered}
\dot{\mathrm{z}}=\left(\delta, \gamma, S / S_{\mathrm{T}}, Q / S_{\mathrm{T}}, \cos \phi, \quad a_{2}, a_{3}, a_{5}, a_{7}, a_{9}, a_{11}, a_{13}, a_{15}, a_{17}, a_{19}\right) \\
\dot{\mathrm{z}} \in D
\end{gathered}
$$

Regard the center value in the subspace as initial point to calculate. The maximum of $\lambda$ and $\mu$ in each subspace will be solved by adopting the parallel algorithm ${ }^{[21]}$ of generalized reduced gradient method (GRG).

Choosing the maximum value in all subspace and the corresponding values of $x$ constitute a point of the maximum function curve.

On caculation in this paper, multidimensional space D could be divided into 42.51 million subspaces. So the objective function is convex function in the subspace of optimal solution.

\section{TECHNICAL ECONOMY ANALYSIS OF THE REACTANCE RATE}

\subsection{Harmonic elimination performance optimal reactance rate $12 \%$}

From the fig. 2 show that the maximum minimum optimization value of reactance rate $x$ within the 
range of $11 \% \sim 13 \%$, and both two max values of the objection function are 1. In other words, the grid parameter $\dot{z}$ within the whole power grid state space $\mathrm{D}, \lambda$ and $\mu$ both are less than or equal to 1 , that means, for this kinds of capacitor banks, only reduce harmonics not amplify harmonics when capacitor banks work $^{[22-23]}$. More importantly, it possess the universal features which adapted to all the substation parameters of $220 \mathrm{kV}$ and $110 \mathrm{kV}$.

\subsection{Analysis of reactance rate application range of $4.3 \% \sim 4.5 \%$}

As shown in Fig. 3 that the suboptimal reactance rate is $4 \%$, but it's likely to lead to resonance degree increases when occur slight change of the reactance rate, without the universal application value.

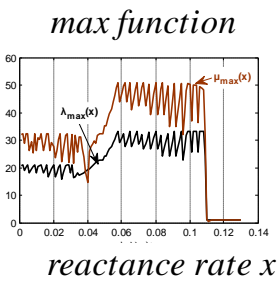

(a)Harmonic current injected into three-winding transformer medium voltage side

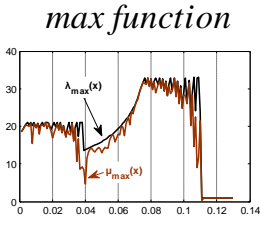

reactance rate $x$

(c)Harmonic current injected into double-winding transformer low voltage side

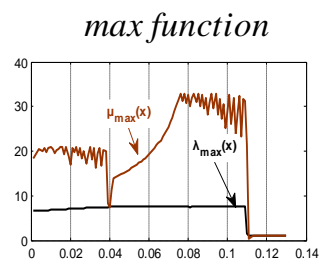

reactance rate $x$

injected into three- winding transformer medium voltage side

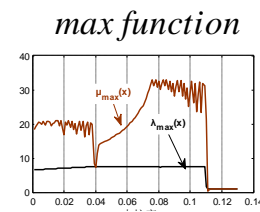

reactance rate $x$

(d) Harmonic current injected into double-winding transformer low voltage side
(b)Harmonic current

Fig. 3 Curves of objective maximum functions while

$\mathrm{S} / \mathrm{ST} \geq 15$ and $\mathrm{Q} / \mathrm{ST} \leq 0.2$

Studies have shown that the transformer shortcircuit ratio $S / S_{\mathrm{T}}$ and capacitor compensation rate $Q / S_{\mathrm{T}}$ have great influence on the objective function. Calculation $\square$ max $(x)$ and $\square \max (x)$ in the new parameters scope of $15 \leq S / S_{\mathrm{T}}$ and $Q / S_{\mathrm{T}} \leq 0.2$ and its curve is shown in fig. 4 . when reactance rate at $3.9 \%$ $\sim 5 \%$, the maximum of objective function will decrease and collapse into a gap that has application value.

Considering the deviation on the manufacture of capacitor components and reactor components impedance, suboptimal reactance rate should be designed to $4.3 \%$, compare with the value of typical reactance rate, it is very close to $4.5 \%$ and very small difference. The numerical results are shown in table 1.

Notice that the value of $\square$ is less than 4.77 when the reactance rate in $4.3 \% \sim 4.5 \%$ in table
1.According to the analysis of $\square$, under the condition that harmonic power quality of the transformer substation is qualified, capacitor element is guaranteed by the safe operation for a long time.

Tab.1 Minimax optimization while $\mathrm{S} / \mathrm{ST} \geq 15$ and $\mathrm{Q} / \mathrm{ST} \leq 0.2$

\begin{tabular}{|c|c|c|c|c|}
\hline $\begin{array}{l}\text { Injection } \\
\text { point of harmoic } \\
\text { sources }\end{array}$ & $\begin{array}{l}\text { Maximum } \\
\text { Target } \\
\text { Function }\end{array}$ & $\begin{array}{l}\text { optimm } \\
\text { point } \\
x=12 \%\end{array}$ & $\begin{array}{l}\text { secondary } \\
\text { point } \\
x=4.3 \sim 4.5 \%\end{array}$ & $x=6 \%$ \\
\hline \multirow{2}{*}{$\begin{array}{l}\text { Three-winding } \\
\text { transformer } \\
\text { medium voltage } \\
\text { side }\end{array}$} & $\max (x)$ & 0.997 & 1.89 & 18.85 \\
\hline & $\operatorname{nax}(x)$ & 0.980 & 4.05 & 26.06 \\
\hline \multirow{2}{*}{$\begin{array}{l}\text { Three-winding } \\
\text { transformer high } \\
\text { voltage side }\end{array}$} & $\max (x)$ & 1.000 & 1.673 & 5.42 \\
\hline & $\max (x)$ & 0.980 & 4.05 & 26.06 \\
\hline \multirow{2}{*}{$\begin{array}{l}\text { Double-winding } \\
\text { transformer low } \\
\text { voltage side }\end{array}$} & $\max (x)$ & 0.997 & 1.775 & 2.48 \\
\hline & max $(x)$ & 0.987 & 1.76 & 2.29 \\
\hline \multirow{2}{*}{$\begin{array}{l}\text { Double-winding } \\
\text { transformer high } \\
\text { voltage side }\end{array}$} & $\max (x)$ & 1.000 & 1.297 & 1.50 \\
\hline & max $(x)$ & 0.988 & 1.76 & 2.48 \\
\hline
\end{tabular}

\subsection{Problem on reactance rate of $6 \%$}

It's usually to adopt to the capacitor group with reactance rate of $6 \%$ for the substation of $220 \mathrm{kV}$ $110 \mathrm{kV}$, but it is not optimized values, especially in the three-winding transformer substation, capacitor group with reactance rate of $6 \%$ exist great resonance.

\subsection{The economic comparison on reactance rate of $12 \%$ and $4.5 \%$}

The bigger the reactance rate $x$ increase,the greater the capacity loss of capacitor is, the rated voltage and rated current of capacitor and reactance will increase to $1 /(1-x)$ times, that increase the cost of manufacture.And as a result of the power loss of reactor is very big, it can't be ignored that the power loss caused by increasing reactance rate.

Comparing with reactance rate of $4.5 \%$ and $12 \%$ and analysing its economic characteristics by the way of life cycle analysis, see appendix C.It can be found that the comprehensive cost of capacitor group, with $12 \%$ reactance rate, is 2.14 times as $4.5 \%$.It will produce a great price to adopt the reactance rate of $12 \%$.

\section{CONCLUSIONS}

The reactance rate of $4.3 \% \sim 4.5 \%$ is benign in technical and economic, the ability of resonance elimination of which is just behind reactance rate in $12 \%$. In a substation with qualified electric energy, the compensation rate of capacitance $\mathrm{Q} / \mathrm{S}_{\mathrm{T}} \geq 0.2$, and 
Short circuit ratio $S / S_{\mathrm{T}} \leq 15$. The rate of $4.3 \% \sim 4.5$ should be applied, which is suitable for almost $220 \mathrm{kV} \sim 110 \mathrm{kV}$ substation.

The reactance rate of $12 \%$ is the best in eliminating resonance, which has universal, while its economic features are not as good as the rate in 4.3\% 4.5\%.In the substation that harmonic power quality exceed the standard, or the compensation rate of capacitance $\mathrm{Q} / \mathrm{S}_{\mathrm{T}}>0.2$, or the short-circuit ratio $\mathrm{S} / \mathrm{ST}<15$, the reactance rate could be designed as $12 \%$.

The reactance rate of $6 \%$ is not as good as the rate of $4.3 \% \sim 4.5 \%$, which neither in eliminating resonance nor the cost of manufacture and application; in the three-winding transformer substation, high strength resonance is still exist in the capacitor banks with $6 \%$ reactance rate; so it's inadvisable to design a $6 \%$ reactance rate in $220 \mathrm{kV}$ $110 \mathrm{kV}$ substation.

\section{REFERENCES}

[1] Angelo Baggini, Zbigniew Hanzelka. Handbook of Power Quality. Italy: John Wiley \& Sons, Ltd, 2008, Annex4.

[2] George J.Wakileh, Power systems harmonics fundametals, analysis and filter design. Beijing, China: China Machine Press, 2005.

[3] Jianming Li. Power harmonic suppression and passive power fiter technology. The World of Power Supply, 2009(2): 21-25.

[4] Shiqing Chen, Xinshang Zhao, Fuxiang Li. Prevention of har-monic amplification in operation of parallel capacitor. High Voltage Engineering, 2004, 30(12): 19-22.

[5] Chengkui Shi. Study on harmonic resonance and harmonic en-largement of power network capacitor bank. Electric Power Au-tomation Equipment, 2001, 21(7) : 3638.

[6] Tao Wu, Jing Xiao. City power grid harmonic analysis and control pol icy. High Voltage Engineering, 2006, 32(10):132-133.

[7] Saiheng Lei, Tiecheng Lu, Bo Zhang, et al. Selection of series reactors in reactive compensation capacitors device in power dis-tribution networks. High Voltage Engineering, 2004, 30(10): 29-31.

[8] Youyuan Tian. Harmonic problems in operation of power system shunt capacitor. Northeast Electric Power Technology, 1996,(2): 1-6.

[9] Feikong Lu, Xuesong Bai, Yu He, et al. Harmonic amplification of capacitors in parallel and countermeasures. Heilongjiang Electric Power, 2003, 25(1):27-29.

[10] Yongjie Yu. Harmonics wave restraining of serial reactor and se-lection of reactance rate. Electrotechnical Application, 2005, 24(3):74-77.

[11] Chiguo $\mathrm{Hu}$, Jing Zhang, Yinyong He. A reactive compensation system with harmonic. Northeast Electric Power Technology, 2005, (6):17-29.

[12] Dan Wu, Jiaoping Kan. Discussion on the prevention from en-largement of high order harmonics wave. High Voltage Engineering, 2002, 28(9): 40-41.

[13] Bosheng Chen. Harmonics restraining function of serial reactor and selection of reactance rate. Power System Technology, 2003, 27(12):92-95.

[14] GB/T 30841-2014 Chinese Standard SN for Books. Beijing, China: China Standard Press, 1993.

[15] Hiyama, T.Hammam $M S A$ A, Ortmeyer $T H$. Distribution system modelling with distributed harmonic sources. IEEE Trans on Power Delivery, 1989, (2): 12971304.

[16] Yong Zhao, Tao Zhang, Jian hua LI, et al. Study on harmonic resonance and harmonic enlargement of power network capacitor bank. Proceedings of the CSEE, 2002, 22(4) : 46-50.

[17] CIGRE Working Group 36-05. AC system modeling for AC filter design - an overview of impedance modeling. Electric, 1996, (164): 133-151.

[18] GB/T 11024.1-2010 Chinese Standard SN for Books. Beijing, China: China Standard Press, 2010.

[19] SD325-89 The department of energy. Beijing:1989

[20] GB/T 14549-1993 Chinese Standard SN for Books. Beijing, China: China Standard Press, 1993.

[21] Byrd, R.H., Mary E., Jorge Nocedal. An interior point algorithm for large-scale nonlinear programming. SIAM Journal on Opti-mization, Vol 9, No. 4.

[22] Jingchang Wu. Harmonics in Power supply system. Beijing: Chinese Electric Power Publishing Company, 1998:43

[23] GB/T 5795-1986 Chinese Standard SN for Books. Beijing, China: China Standard Press, 1986. 